\title{
EVALUATION OF SALIVARY AND SERUM VISFATIN IN ORAL BULLOUS EROSIVE LICHEN PLANUS AND ORAL SQUAMOUS CELL CARCINOMA
}

\author{
Wesam Abdel Moneim*, Amira Maged ${ }^{* *}$ and Olfat Shaker ${ }^{* * *}$
}

\begin{abstract}
Introduction: Bullous Erosive Oral Lichen Planus (BEOLP) is a potentially malignant condition with high rate of transformation into Oral Squamous Cell Carcinoma (OSCC), and aiming to reduce the morbidity and mortality rate arising from OLP; the early diagnosis of oral cancer was recommended. Many biomarkers were found to be released into the serum and the saliva during the course of both diseases, BEOLP and OSCC. One of these proteins is visfatin.
\end{abstract}

Aim of the study: The aim of this study was to evaluate salivary level of visfatin and to find out if it can be used instead of its serum level as an early diagnostic biomarker for patients with either BEOLP or OSCC.

Subjects and Methods: The study was conducted on 3 groups; group (A) which included fifteen medically free subjects, group (B) which included fifteen patients suffering from BEOLP, and finally group (C) which included fifteen patients suffering from OSCC. Serum and salivary samples were collected and visfatin levels were measured using an ELISA kit (Enzyme Linked Immuno Sorbent Assay). Data were analyzed by SPSS.

Results: The present study detected no statistically significant difference between mean visfatin levels in BEOLP and OSCC groups; both showed statistically significantly higher mean levels than control group which showed the lowest mean visfatin level. It Also revealed a statistically significant positive (direct) correlation between serum and salivary visfatin levels $(r=0.835, P$-value $<0.001)$ i.e. an increase in serum level of visfatin is associated with an increase in salivary level of Visfatin.

Conclusion: Salivary level of visfatin can be used instead of its serum level as a biomarker for BEOLP and OSCC.

KEYWORDS: Visfatin, BEOLP, OSCC.

\footnotetext{
*Asso.Professor at Department of Oral Medicine, Periodontology and Diagnosis, Faculty of Oral and Dental Medicine, Cairo University. ** Lecturer at Department of Oral Medicine, Periodontology and Diagnosis, Faculty of Oral and Dental Medicine, Cairo University. ***Prof. at Department of Medical Biochemistry, Faculty of Medicine, Cairo University.
} 


\section{INTRODUCTION}

Visfatin, which is also known as pre-B-cell colony enhancing factor and nicotinamide phosphoribosyl-transferase, was originally cloned by Samal et al. in 1994. ${ }^{(1)}$ In addition to adipose tissue, this protein is synthesized by various cell types and tissues, comprising dendritic cells and peripheral blood monocytes. (2) Furthermore, visfatin is usually expressed by lymphocytes, bone marrow, liver cells, skeletal muscles, trophoblasts, and fetal membranes. ${ }^{(2)}$

This pleiotropic mediator acts as a growth factor, cytokine, and enzyme. ${ }^{(3)}$ In addition; it has several pro-inflammatory roles. It can encourage the expression of interleukin 6 (IL-6), IL-1 $\beta$ and tumor necrosis factor alpha (TNF- $\alpha$ ) in human monocytes. Contrariwise, an increase in the levels of the above mentioned pro-inflammatory cytokines in the periodontal tissues can induce visfatin production. Likewise, it has been validated that the activation of T-cells, chemotaxis of CD4+ monocytes and CD19+ B-cells and inhibition of neutrophil apoptosis can be affected by visfatin. Several studies have indicated the elevation of visfatin levels in inflammatory diseases as for example rheumatoid arthritis, type 2 diabetes mellitus and inflammatory bowel disease. ${ }^{(2,4)}$ Visfatin was found to be one of the adipokines that may play a role in the pathogenesis and severity of psoriasis in addition to its role in diseases of the cardiovascular system. ${ }^{(5)}$

Moreover, visfatin has been proposed as being expressed in normal, inflamed, and tumor tissues. $(6,7)$ It possesses nicotinamide adenine dinucleotide (NAD) biosynthetic activity and regulates growth, angiogenesis, and apoptosis in mammalian cells. $(8,9)$ Apoptotic cell death was thought to be a contributory cause of basal cell destruction in oral lichen planus (OLP). ${ }^{(10)}$

Additionally, bullous erosive oral lichen planus (BEOLP) is considered a potentially malignant condition, and aiming to reduce the morbidity and mortality rate arising from oral lichen planus; the early diagnosis of oral cancer was recommended. (11) It was found that visfatin is identifiable and measurable in saliva. ${ }^{(12)}$

Determining the potential for malignant transformation of oral lichen planus (OLP) is complicated by difficulties in diagnosis. ${ }^{(13)}$

Numerous studies have reported that the inflammatory infiltrate can be a strong risk factor for cancer development in chronic, inflammatory conditions. Adipose tissue produces several proteins (adipocytokines), such as TNF- $\alpha$, IL-6, type-1 plasminogen activator inhibitor (PAI-1), adipo-nectin, leptin, resistin, visfatin and apelin are associated with the risk of cancer at various sites (e.g., breast, prostate gland, endometrium and colo-rectum). ${ }^{(14,15)}$

The altered secretion of metabolically active, proinflammatory adipo-cytokines from adipose tissue is believed to play a key role in the mechanisms related to cancer. ${ }^{(16)}$

To the authors' knowledge, there have been no studies that investigated salivary concentrations of visfatin in BEOLP and OSCC. The aim of this study was to evaluate salivary level of visfatin and to find out if it can be used instead of its serum level as an early diagnostic biomarker for patients with either BEOLP or OSCC.

\section{SUBJECTS\& METHOD:}

\section{Study population:}

The present study was performed on a total of 45 subjects, 13 males and 32 females. All participants were recruited from the outpatient clinic of Oral Diagnosis, Oral Medicine and Periodontology Department, Faculty of Oral and Dental Medicine, Cairo University.

\section{Inclusion criteria:}

Participants were systemically free as evaluated by the aid of Dental modification of the Cornell medical index to standardize their systemic 
condition. ${ }^{(17)}$ (Brightman, 1994). They had no history, symptoms, and/or signs of infection and allergy. Furthermore, they were selected to be free of any oral lesions other than OSCC or BEOLP which had to have the following criteria: (i) painful lesions (ii) no topical treatment for 2 weeks, and no systemic treatment used for BEOLP for one month before the start of sampling.

\section{Exclusion criteria:}

Pregnant or breast-feeding women or children patients, those who use corticosteroids or other immune-suppressive drugs, and patients who had allergy to macrolide antibiotics and smokers, all were excluded from the study.

\section{Ethical procedures:}

All subjects were informed about the detailed procedure and they were given written approval consent to sign. Patients were treated after the samples had been collected. The study was performed between September 2014 and February 2016.

The forty five selected participants were divided into three groups as follows:-

\section{Group A :}

It included 15 medically free subjects, 4 males and 11 females. Their ages ranged from 35-42 years.

\section{Group B:}

It included 15 patients suffering from BEOLP, 3 males and 12 females. Their ages ranged from $36-48$ years.

\section{Group C:}

It included 15 patients suffering from OSCC, 6 males and 9 females. Their ages ranged from 41-58 years.

\section{II- Clinical monitoring}

The following procedures were performed for all individuals included in the study:

\section{Saliva collection}

Un-stimulated whole saliva was collected from all participants. Each individual was requested to abstain from eating, drinking and brushing his/her teeth for at least $60 \mathrm{~min}$ prior to collection. Saliva samples were collected between 8 a.m. and 2 noons. Un-stimulated whole saliva was collected using the drooling technique. Each subject rinsed their mouth with water before saliva collection, and then the subject was asked to swallow to remove saliva from the mouth. The subject was seated upright, and leaned his/her head forward over a test tube with a funnel, allowing the saliva to drain into the tube. Whole saliva $(\sim 5 \mathrm{~mL})$ was obtained from each individual. During saliva collection, the test tube was placed on ice. At the end of the collection, any remaining saliva in the patient's mouth was expelled into the test tube.

\section{Serum collection}

Venous samples were obtained to measure the serum visfatin level using the ELISA technique after 12 hours of fasting. Blood samples were collected in sterile tubes and allowed to clot at room temperature. Sera were isolated by centrifugation and stored frozen below -20 until assayed for the visfatin level.

\section{3-Saliva processing}

Saliva obtained was centrifuged at $15,000 \times \mathrm{g}$ (MPW-65R, MPW, Med Instrument, Warszawa, Poland) for $15 \mathrm{~min}$ at $4^{\circ} \mathrm{C}$ to remove insoluble material. Supernatant was divided into 1-mL aliquots in pre-chilled cryo-tubes. The specimens were immediately frozen $\left(-80^{\circ} \mathrm{C}\right)$ until analysis.

\section{4- Visfatin level detection by ELISA:}

The level of visfatin was measured in serum and saliva samples by using ELISA kit provided by $E I A a b^{\circledR}$, China Catalog No: E0638h. The ELISA is based on the competitive binding Enzyme Linked Immune Sorbent Assay technique. The micro-titre 
plate provided in this kit has been pre-coated with an antibody specific to $\mathrm{C} 4 \mathrm{a}, \mathrm{C} 4 \mathrm{a}$ in the sample or standard competes with a fixed amount of biotinlabeled C4a for sites on a pre-coated Monoclonal antibody specific to $\mathrm{C} 4 \mathrm{a}$. Then Avidin conjugated to Horseradish was added to each well and incubated. Then a TMB substrate solution was added. Then termination was done by the addition of sulphuric acid solution and the color change was measured spectro-photo-metrically at a wavelength of $450 \mathrm{~nm}$ $\pm 2 \mathrm{~nm}$. The concentration of $\mathrm{C} 4 \mathrm{a}$ in the samples was then determined by comparing the O.D. of the samples to the standard curve.

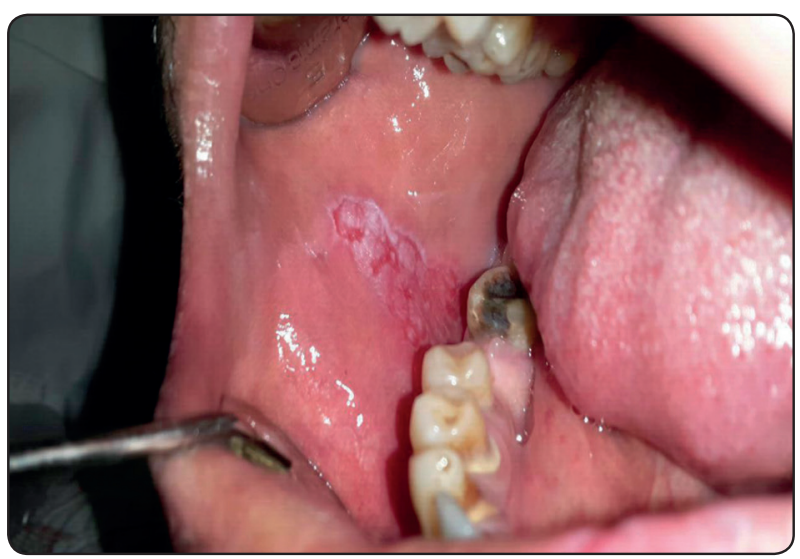

Fig. (1) A clinical photograph showing BEOLP in right side of cheek mucosa.

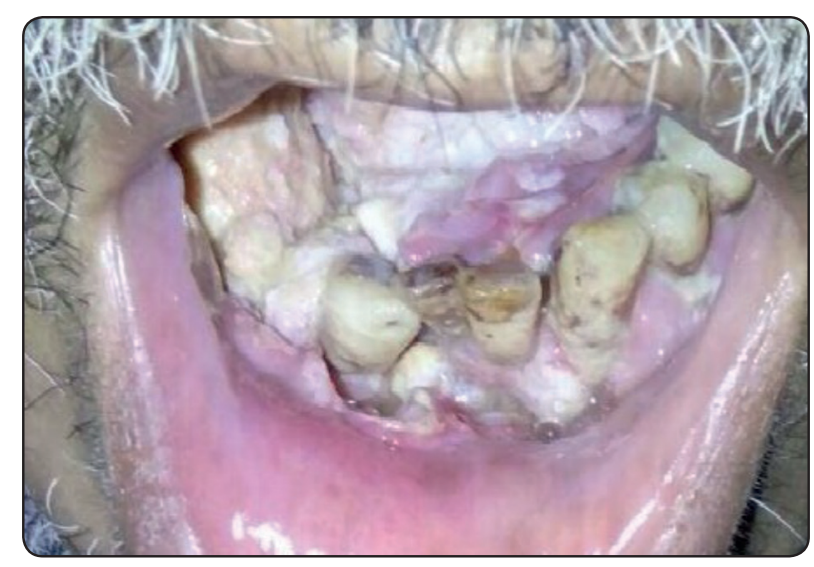

Fig. (2) A clinical photograph showing OSCC in floor of mouth.

\section{Statistical Analysis}

Quantitative data were presented as mean, median, standard deviation (SD), range (Minimum - Maximum) and 95\% Confidence interval (95\% CI) for the mean values. Data were explored for normality by checking the data distribution and using Kolmogorov-Smirnov and Shapiro-Wilk tests. Visfatin levels in serum showed parametric distribution while visfatin levels in saliva showed non-parametric distribution.

For parametric data; one-way ANOVA followed by Tukey's test were used to compare between the three groups. For parametric data; one-way ANOVA followed by Tukey's test were used to compare between the three groups. For nonparametric data; Kruskal-Wallis test was used to compare between the three groups. Mann-Whitney U test with Bonferroni's adjustment was used for pair-wise comparisons when Kruskal-Wallis test is significant. Spearman's correlation coefficient was used to determine the correlation between visfatin levels in serum and saliva.

ROC (Receiver Operating Characteristic) curve was constructed to determine the cut-off values of VISFATIN for detection of BEOLP. Areas under the ROC curve (AUCs), sensitivity, specificity, predictive values and diagnostic accuracy was calculated.

The significance level was set at $\mathrm{P} \leq 0.05$. Statistical analysis was performed with IBM $^{\circledR}$ SPSS $^{\circledR}$ Statistics Version 20 for Windows.

\section{RESULTS}

\section{Demographic data}

There was no statistically significant difference between mean age values in the three groups. There was also no statistically significant difference between gender distributions in the three groups.

( ${ }^{8}$ IBM Corporation, NY, USA.

() SPSS, Inc., an IBM Company. 
TABLE (1): Descriptive statistics and results of oneway ANOVA and Chi-square tests for comparison between demographic data in the three groups

\begin{tabular}{|l|c|c|c|c|}
\hline & \multicolumn{3}{|c|}{ OSCC } & \multicolumn{2}{c|}{ BEOLP } & Control & $P$-value \\
\hline $\begin{array}{l}\text { Age (mean, } \\
\text { SD) }\end{array}$ & $\begin{array}{c}47.7 \\
(14.1)\end{array}$ & $42.3(11.0)$ & $\begin{array}{c}38.5 \\
(7.8)\end{array}$ & 0.095 \\
\hline $\begin{array}{c}\text { Gender (n, \%) } \\
\text { Male }\end{array}$ & $6(40.0)$ & $3(20.0)$ & $4(26.7)$ & 0.469 \\
Female & $9(60.0)$ & $12(80.0)$ & $11(73.3)$ & \\
\hline
\end{tabular}

*: Significant at $P \leq 0.05$

\section{Visfatin levels}

Whether in serum or saliva, there was no statistically significant difference between mean visfatin levels in OSCC and BEOLP groups; both showed statistically significantly higher mean levels than control group which showed the lowest mean visfatin level.

\section{Correlation between serum and salivary visfatin levels}

There was a statistically significant positive (direct) correlation between serum and salivary visfatin levels ( $r=0.835, P$-value $<0.001)$ i.e. an increase in serum level of visfatin is associated with an increase in salivary level of visfatin.

TABLE (2): Descriptive statistics and results of one-way ANOVA, Tukey's tests, Kruskal-Wallis and MannWhitney $\mathrm{U}$ tests for comparison between visfatin levels in the three groups

\begin{tabular}{|c|c|c|c|c|c|c|c|c|c|}
\hline & \multirow{2}{*}{ Group } & \multirow{2}{*}{ Mean } & \multirow{2}{*}{ SD } & \multirow{2}{*}{ Median } & \multirow{2}{*}{ Minimum } & \multirow{2}{*}{ Maximum } & \multicolumn{2}{|c|}{$95 \% \mathrm{CI}$} & \multirow{2}{*}{$P$-value } \\
\hline & & & & & & & Lower bound & Upper bound & \\
\hline \multirow{3}{*}{ E } & OSCC & $34.5^{\mathrm{a}}$ & 7.0 & 32.6 & 22.8 & 51.3 & 30.6 & 38.4 & \multirow{3}{*}{$<0.001 *$} \\
\hline & BEOLP & $36.4^{\mathrm{a}}$ & 5.7 & 35.5 & 29.7 & 50.1 & 33.2 & 39.5 & \\
\hline & Control & $13.8^{\mathrm{b}}$ & 2.3 & 13.0 & 10.9 & 18.3 & 12.5 & 15.1 & \\
\hline \multirow{3}{*}{ 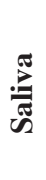 } & OSCC & $2184.7^{\mathrm{a}}$ & 898.0 & 2018.0 & 1068.0 & 3681.0 & 1687.5 & 2682.0 & \multirow{3}{*}{$<0.001 *$} \\
\hline & BEOLP & $2133.5^{\mathrm{a}}$ & 570.0 & 2047.0 & 1236.0 & 3247.0 & 1817.9 & 2449.2 & \\
\hline & Control & $160.1^{\mathrm{b}}$ & 24.9 & 159.0 & 117.0 & 191.0 & 146.3 & 173.9 & \\
\hline
\end{tabular}

*: Significant at $P \leq 0.05$, Different superscripts in the same column are statistically significantly different

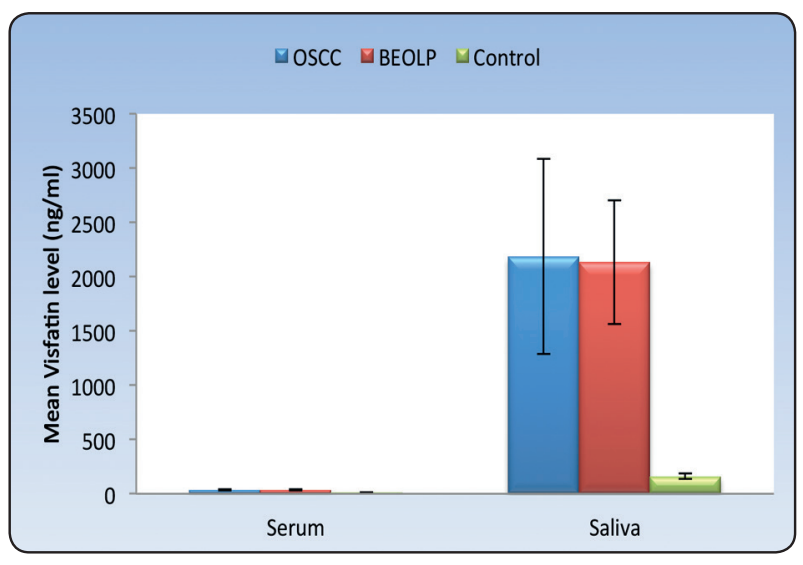

Fig. (3): Bar chart representing mean and standard deviation values of visfatin levels in the three groups

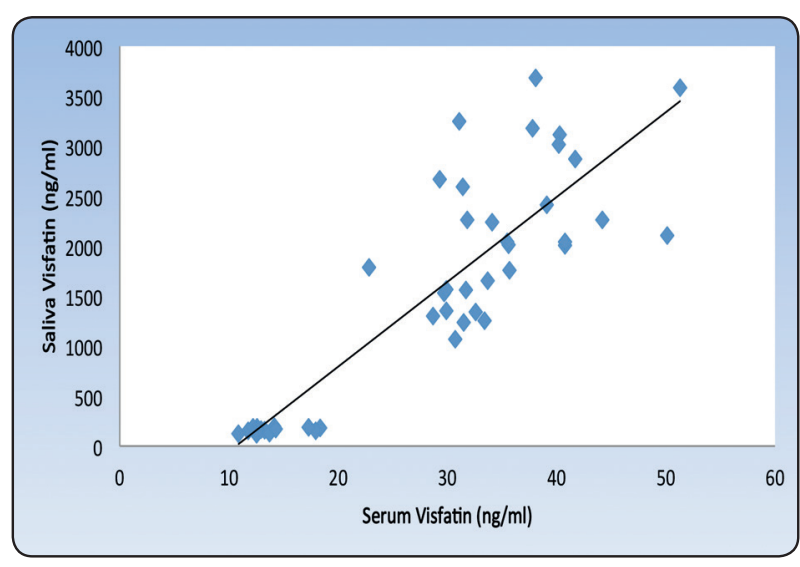

Fig. (4): Scatter diagram representing positive correlation between serum and salivary levels of visfatin 


\section{ROC curve analysis (Cut-off values)}

Results of ROC curve analysis are presented in Table (3) and Figures (5-6).

ROC curve analysis of serum and salivary levels of visfatin in the present study showed cut-off values of 18.3 and $191.0 \mathrm{ng} / \mathrm{ml}$, respectively. At these cutoff values, the diagnostic accuracy of visfatin as a marker for detecting OSCC and BEOLP is $100.0 \%$.

TABLE (3): Sensitivity, specificity, predictive values, diagnostic accuracy, Area under the ROC curve (AUC), 95\% confidence interval (95\% CI) of serum and salivary visfatin levels

\begin{tabular}{|c|c|c|c|c|c|c|c|c|}
\hline $\begin{array}{c}\text { Visfatin } \\
\text { (ng/ml) }\end{array}$ & Cut-off & Sensitivity \% & Specificity \% & $\mathbf{+ P V} \%$ & $\mathbf{- P V} \%$ & $\begin{array}{c}\text { Diagnostic } \\
\text { accuracy } \%\end{array}$ & AUC & $\mathbf{9 5 \%} \mathbf{C I}$ \\
\hline Serum & 18.3 & 100.0 & 100.0 & 100.0 & 100.0 & 100.0 & 1.000 & $0.884-1.000$ \\
Saliva & 191.0 & 100.0 & 100.0 & 100.0 & 100.0 & 100.0 & 1.000 & $0.884-1.000$ \\
\hline
\end{tabular}

+PV: Positive Predictive Value, -PV: Negative Predictive Value

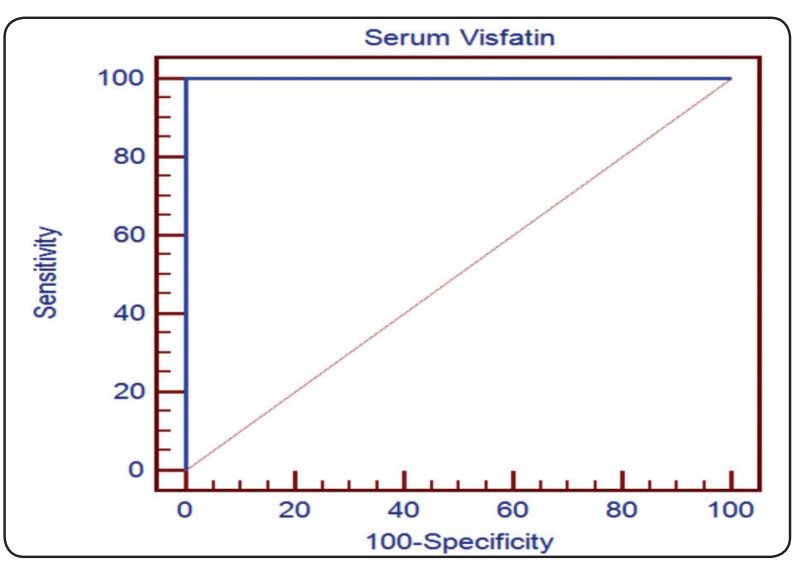

Fig. (5): ROC curve for Visfatin level in serum.

\section{DISCUSSION:}

The aim of this study was to evaluate salivary level of visfatin and to find out if it can be used instead of its serum level as an early diagnostic biomarker for patients with either BEOLP or OSCC.

Salivary samples were chosen for evaluation, as saliva is considered a diagnostic material, ${ }^{(18)}$ abundant and has a simple sampling technique, fast and non-invasive; also, has no need for specialized equipment for its sampling. ${ }^{(19,20)}$

The present study showed no statistically

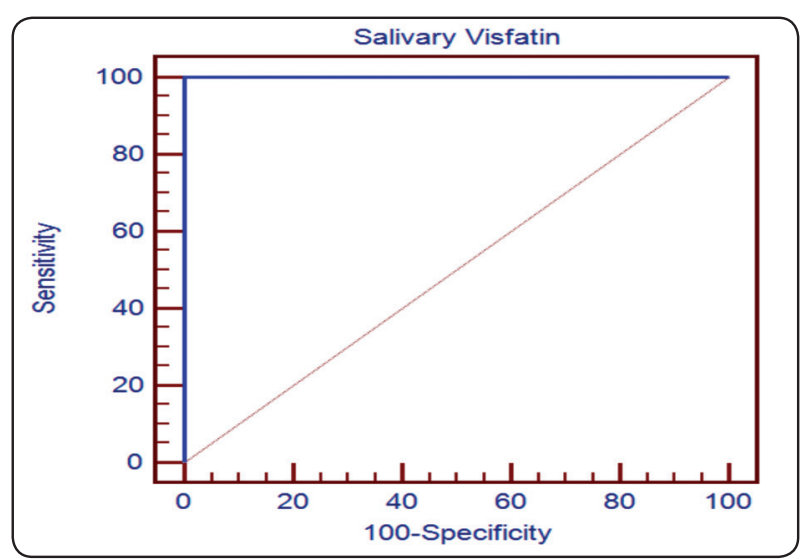

Fig. (6): ROC curve for Visfatin level in saliva.

significant difference between both sex and mean age values in the three groups, as shown in table (1). These results are not in agreement with the study performed by Eisen in 2003. ${ }^{(21)}$ His study detected that both OLP and OSCC were more common in females and, on average, being in the sixth decade of life similar to the results of Fitzpatrick et al in 2014. ${ }^{(13)}$

Regarding the mean visfatin levels, whether in serum or saliva, the present study showed no statistically significant difference between mean visfatin levels in OSCC and BEOLP groups; 
however, both showed statistically significantly higher mean levels than control group which showed the lowest mean visfatin level. These results are in agreement with Tabari et al in $2014^{(22)}$ who found that salivary visfatin levels were significantly higher in the patients with $\mathrm{CP}$ (chronic periodontitis) than healthy controls. In addition, a positive and significant correlation was found between CAL (clinical attachment level) loss and salivary concentrations of visfatin. They explained their results by the role of visfatin in the pathogenesis of periodontal disease by local immune-inflammatory reactions in periodontal tissues that can result in degradation and resorption of the periodontal structures, respectively. Also, the present results are similar to the study performed by Özcan et al in 2015. ${ }^{(23)}$ They demonstrated higher salivary visfatin levels in patients with gingivitis and periodontitis compared to those of healthy subjects.

Moreover, these results are in accordance with

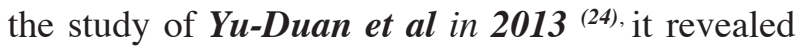
that visfatin serum concentrations were significantly elevated in a fully adjusted model in patients with OSCC. Furthermore, they found that visfatin was gradually increased with stage progression .This could be attributed to the role of visfatin in activation of leukocytes and hence production of cytokines such as IL-1 $\beta$, IL-6 and tumor necrosis factor. Therefore, visfatin could be regarded as an adipo-cytokine with pro-inflammatory, immunemodulating, and apoptosis-inhibiting potentials. ${ }^{(3)}$

Similarly, Badran et al in $2014^{(5)}$ found that the serum level of visfatin was statistically significantly higher in patients suffering of psoriasis than in the healthy control group. Moreover, Ismail \& Mohamed in $2012^{(25)}$ detected that serum visfatin level was significantly higher in patients with severe psoriasis than those with mild and moderate psoriasis with no statistically significant difference between normal weight, overweight, and obese patients in the serum level of visfatin. On the same hand, Gerdes et al ${ }^{(26)}$ found that serum visfatin is increased in patients with psoriasis independent of other factors such as basal metabolic index (BMI).

The present study detected a statistically significant positive (direct) correlation between serum and salivary levels of visfatin i.e. an increase in serum level of visfatin is associated with an increase in salivary level of visfatin.

To author's knowledge, little studies were performed about the salivary level of visfatin in oral diseases. However, these results are similar to the study performed by Tabari et al, ${ }^{(22)}$ who concluded that salivary concentration of visfatin could be a beneficial and convenient alternative for serum and GCF visfatin in the evaluation of periodontal disease.

On the same hand, Abolfazli et al in $2015^{\text {(27) }}$ demonstrated higher level of visfatin in the saliva more than in serum and this could be explained by the leakage of saliva from salivary glands, and they considered it as a biomarker that reflects the function of periodontal tissues.

The diagnostic accuracy of visfatin measurements as a salivary marker was evaluated by the ROC plot method that proved to have very high sensitivity, specificity, and diagnostic accuracy. Actually, it has been suggested that a test's diagnostic accuracy should not be lower than $80 \%$ to be considered applicable, valid and suitable for diagnostic purposes. ${ }^{(28)}$ In ROC analysis, an AUC (area under curve) value denotes the combined effects of both specificity and sensitivity for an assay system. The present study determined the AUC values in both serum and saliva as 1.000 , and 1.000 , respectively. It is known that an AUC value of 0.9 or higher refers to an excellent diagnostic marker. ${ }^{(29)}$

For using saliva as a diagnostic material (18), different candidate biomarkers for the assessment of periodontal disease were investigated; in addition, several studies have demonstrated that 
adipose tissue secretes adipo-cytokines, which are mediators that have important roles in immunity and inflammation. ${ }^{(4)}$

Moreover, as quite a lot of studies have testified the elevation of visfatin level in many inflammatory illnesses such as Behcet syndrome, chronic kidney disease, chronic obstructive pulmonary disease, appendicitis, metabolic syndrome ${ }^{(3,30,31)}$ rheumatoid arthritis, type II diabetes mellitus and inflammatory bowel disease. ${ }^{(2,4)}$

On the same hand, visfatin is considered as a risk factor for cardiovascular diseases. ${ }^{(32,33)}$ Also, an increase in the expression of visfatin by macrophages in unstable atherosclerotic lesions indicates the possible role of visfatin in destabilization of the atherosclerotic plaque ${ }^{(34)}$

Accordingly, it can be concluded that salivary level of visfatin can be used over its serum level as a biomarker for OSCC and chronic inflammatory and immune modulating conditions such as BEOLP.

\section{REFERENCES}

1. Samal B, Sun Y, Stearns G, Xie C, Suggs S, McNiece I Cloning and characterization of the cDNA encoding a novel human pre-B-cell colony-enhancing factor. Mol Cell Biol 1994; 14:1431-1437.

2. Fukuhara A, Matsuda M, Nishizawa M, Segawa K, Tanaka M, Kishimoto K, Matsuki Y, Murakami M. Visfatin: a protein secreted by visceral fat that mimics the effects of insulin. Science. 2005; 307:426-30.

3. Moschen AR, Kaser A, Enrich B, Mosheimer B, Theurl $\mathrm{M}$, Niederegger $\mathrm{H}$, et al. Visfatin, an adipocytokine with proinflammatory and immunomodulating properties. J Immunol. 2007; 178:1748-58.

4. Tilg $\mathrm{H}$ and Moschen AR. Role of adiponectin and PBEF/ visfatin as regulators of inflammation: involvement in obesity-associated diseases. ClinSci (Lond) 2008; 114(4):275-88.

5. Badran FK, Genedy RM, Swelem RS, Al-Rawi MZ. Evaluation of serum level of visfatin among psoriatic patients. 2014; Volume: 34, Issue: 2, Page: 107-113
6. Bae SK, Kim SR, Kim JG, Kim JY, Koo TH, Jang HO. Hypoxic induction of human visfatin gene is directly mediated by hypoxiainducible factor-1. FEBS Lett. 2006; 580:4105-13.

7. Gen R, Akbay E, Muslu N, Sezer K, Cayan F. Plasma visfatin level in lean women with PCOS: relation to proinflammatory markers and insulin resistance. GynecolEndocrinol. 2009; 25:241-5.

8. Bi TQ, Che XM. Nampt/PBEF/visfatin and cancer. Cancer Biol. Ther. 2010; 10:119-25.

9. Galli M, Van-Gool F, Rongvaux A, Andris F, Leo O. The nicotinamidephosphoribosyltransferase: a molecular link between metabolism, inflammation, and cancer. Cancer Res. 2010; 70:8-11.

10. Neppelberg E, Johannessen AC, Jonsson R. Eur J Oral Sci. 2001 Oct; 109(5):361-4.Apoptosis in oral lichen planus.

11. Mattasson U, Mats Jotell, PalleHolmstrup: Oral Lichen Planus and malignant transformation: Is a recall of patients justified?C review in Oral Bio \& Med 2002, vol. 13 no.5390-396.

12. Mamali I, Roupas ND, Armeni AK, Theodoropoulou A, Markou KB, Georgopoulos NA. Measurement of salivary resistin, visfatin and adiponectin levels. Peptides 2012; $331: 120-124$

13. Fitzpatrick SG, Stanley A. Hirsch and Sara C. Gordon. The malignant transformation of oral lichen planus and oral lichenoid lesions: A systematic review JADA 2014; 145(1):45-56.

14. Nakajima TE, Yamada Y, Hamano T, Furuta K, Gotoda T, Katai H, et al. Adipocytokine levels in gastric cancer patients: resistin and visfatin as biomarkers of gastric cancer. J Gastroenterol. 2009; 44:685-90.

15. Sun CA, Wu MH, Chu CH, Chou YC, Hsu GC, Yang T, et al. Adipocytokineresistin and breast cancer risk. Breast Cancer Res Treat. 2010; 123:869-76.

16. Howard JM, Pidgeon GP, Reynolds JV. Leptin and gastrointestinal malignancies. Obes Rev. 2010; 11:863-74.

17. Brightman VJ: Rational procedure for diagnosis and medical risk assessment. In: Burket's Oral medicine, Diagnosis and Treatment. Eds.: Lynch MS, Brightman VJ and Greenberg MS. Lippinocott Company, Philadelphia, Pp. 729-63, 1994. 
18. Gheren LW, Cortelli JR, Rodrigues E, Holzhausen M, Saad WA. Periodontal therapy reduces arginase activity in saliva of patients with chronic periodontitis. ClinOralInvestig. 2008; 12(1):67-72.

19. Gursoy UK, Könönen E, Uitto VJ, Pussinen PJ, Hyvärinen K, Suominen-TaipaleL, et al. Salivary interleukin-1beta concentration and the presence of multiple pathogens in periodontitis. J ClinPeriodontol. 2009;36(11):922-27.

20. Tabari ZA, Azadmehr A, Tabrizi MA, Hamissi J, Ghaedi FB. Salivary soluble receptor activator of nuclear factor kappa B ligand/osteoprotegerin ratio in periodontal disease and health. J Periodontal Implant Sci. 2013; 43(5):227-32.

21. Eisen D. The clinical manifestations and treatment of oral lichen planus. Dermatol. Clin. 2003; 21(1):79-89.

22. Tabari ZA, Abbas Azadmehr, $\dagger$ Ali Nohekhan,\$ NimaNaddafpour,* and FatemehBaharakGhaedi. Salivary Visfatin Concentrations in Patients with Chronic Periodontitis: J Periodontol • August 2014. Vol 85

23. Özcan E, Saygun NI, Serdar MA, Kurt N. Clin Oral Investig.Evaluation of the salivary levels of visfatin, chemerin, and progranulin in periodontal inflammation.2015 May; 19(4):921-8. doi: 10.1007/s00784-014-1308-0. Epub 2014 Aug 28.

24. Yu-Duan T, Wang Chao-Ping, Chen Chih-Yu , Lin LiWen, Lin Tsun-Mei, Hsu Chia-Chang, Chung Fu-Mei, Lin Hsien-Chang, Hsu Hsia-Fen, Lee Yau-Jiunn, HoungJerYiing. Med Oral Patol Oral Cir Bucal. 2013 Mar 1; 18 (2):e180-6.

25. Ismail SA and Mohamed SA. Serum levels of visfatin and omentin-1 in patients with psoriasis and their relation to disease severity. Br J Dermatol 2012; 167: 436-439.

26. Gerdes S, Osadtschy S, Rostami-Yazdi M, Buhles N, Weichenthal M, Mrowietz U. Leptin, adiponectin, visfatin and retinol-binding protein-4 - mediators of comorbidities in patients with psoriasis? ExpDermatol 2012; 21: 43-47.
27. Abolfazli N, SaharJabali, Faribasaleh Saber, ZohrehBabaloo, and AdilehShirmohammadi. Effect of non-surgical periodontal therapy on serum and salivary concentration of visfatin in patients with chronic periodontitis. J Dent Res Dent Clin Dent Prospects. 2015; 9: 11-17.

28. Zweig MH, Campell G. Receiver-operating characteristics (ROC) plots: a functional evaluation tool in clinical medicine.Clin Chem 1993; 39: 929-35.

29. Hanley JA, McNeil BJ. The Meaning and Use of the Area Under a Receiver Operating Characteristic (ROC) Curve. Radiology 1982; 143: 29-36.

30. Axelsson J, Witasp A, Carrero JJ, et al. Circulating levels of visfatin/pre-B- cell colony-enhancing factor 1 in relation to genotype, GFR, body composition and survival in patients with CKD. Am J Kidney Dis 2007; 49:237-244.

31. Brentano F, Schorr O, Ospelt C, et al. Pre-B cell colony enhancing factor/visfatin, a new marker of inflammation in rheumatoid arthritis with pro-inflammatory and matrixdegrading activities. Arthritis Rheum 2007; 56:2829-2839.

32. Liu SW, Qiao SB, Yuan JS, Liu DQ. Association of plasma visfatin levels with inflammation, atherosclerosis and acute coronary syndromes (ACS) in humans. ClinEndocrinol. 2009; 71: 202-7. doi: 10.1111/j.1365 2265.2008.03453.)

33. Romacho T, Azcutia V, Vazquez-Bella M, Matesanz N, Cercas E, Nevado J. et al. Extracellular PBEF/NAMPT/ visfatin activates pro-inflammatory signaling in human vascular smooth muscle cells through nicotinamidephosphoribosyltransferase activity. Diabetologia. 2009; 52:2455-63. doi: 10.1007/s00125-009- 1509-2.).

34. Dahl TB, Yndestad A, Skjelland M, Oie E, Dahl A, Michelsen A, Damas JK, Tunheim SH, Ueland T, Smith C, Bendz B, Tonstad S. Increased expression of visfatin in macrophages of human unstable carotid and coronary atherosclerosis: possible role in inflammation and plaque destabilization. Circulation. 2007; 115:972-80.doi: 10.1161/ circulationaha.106.665893). 